\title{
La hiperostosis esquelética idiopática difusa (DISH) como expresión de un proceso sistémico
}

\author{
Alberto Omar Orden \\ Servicio de Reumatología, Hospital Aeronáutico Central, Buenos Aires, Argentina.
}

La Hiperostosis Esquelética Idiopática Difusa es una condición caracterizada por la calcificación y/u osificación de los tejidos blandos, principalmente entesis, ligamentos y cápsulas articulares. En 1950, Forestier y Rotés-Querol publicaron una serie denominándola "hiperostosis anquilosante vertebral senil" y la distinguieron como entidad nosológica separada de la espondiloartrosis y la espondilitis anquilosante ${ }^{1}$.

En 1975, Resnick publica un extenso estudio que expone su elevada prevalencia, las localizaciones extraespinales y propone el nombre actualmente utilizado de "Difusse Idiopathic Skeleletal Hiperostosis" (DISH)².

El lugar clásico del compromiso es la columna vertebral, siendo la osificación de los tejidos blandos, especialmente del ligamento prevertebral anterior de la columna dorsal, su rasgo característico. La osificación del ligamento longitudinal posterior (OPLL) de la columna, más frecuente en japoneses y otras poblaciones asiáticas, acompaña al DISH hasta en el $50 \%$ de los casos y se la considera como una variante ${ }^{3}$.

Sin embargo, DISH no se limita a la columna vertebral, y puede afectar múltiples sitios periféricos de forma independiente. Las osificaciones de entesis extraespinales son frecuentes, y la observación de su presencia aislada puede conducir al diagnóstico de $\mathrm{DISH}^{4,5}$. Se ha descrito la coexistencia de esta entidad con espondilitis anquilosante ${ }^{6}$.

Inicialmente, Forestier y Rotés-Querol consideraron al DISH como una alteración radiológica "spectacular but inocuous" . Progresivamente, se fueron describiendo cuadros de rigidez espinal de grado variable, síndromes causados por la sobrecarga dinámica de los segmentos móviles, síndromes producidos por ocupación de espacio (compresión del esófago, tráquea, laringe, o médula espinal por el compromiso del canal, con o sin fracturas vertebrales $)^{8-12}$. Por último, la hiperostosis es causa de manifestaciones en estructuras periféricas como hombros, caderas y pies $^{7}$.

La prevalencia global, de acuerdo a estudios realizados en diversas poblaciones o países (incluyendo Finlandia, Budapest, Israel, Corea, Nápoles, los Países Bajos, Sudáfrica y EE.UU.), es muy variable, desde $3,6 \%$ a un $28 \%$, en función de los criterios de diagnóstico o de las poblaciones de estudio. En los estudios radiológicos se ha observado en el 2,4 al 5,4\% de los pacientes mayores de 40 años y en el $11,2 \%$ en los mayores de 70 años, siendo esto confirmado en series necrópsicas. La prevalencia aumenta con la edad, así, en hombres de un $10 \%$ a la edad de 50 a 54 años hasta un 36\% a la edad mayor de 75 años, mientras que en la mujer de un $1,9 \%$ a la edad de 50 a 54 años hasta un $26 \%$ a la edad mayor de 75 años. La relación hombre/ mujer es $2 / 1$, diferencia que disminuye con la edad ${ }^{12-21}$.

No existen criterios clasificatorios definitivos, basándose en general en los planteados por Resnick:

a. La presencia de osificaciones exuberantes en la cara antero lateral (dos si se encuentran en la columna torácica inferior o tres en otras localizaciones),

b. La presencia de una relativa preservación del espacio intervertebral en el segmento afectado y la ausencia de cambios degenerativos en el disco (fenómeno de vacío o esclerosis del cuerpo vertebral), $\mathrm{y}$

c. La ausencia de afectación de las articulaciones

\section{Correspondencia}

Dr. Alberto Omar Orden, Lascano 3518 P.B. “1”, CP 1417, Buenos Aires, Argentina.

E-mail: aoorden@intramed.net.ar

Conflicto de intereses. El autor declara no tener ningún conflicto de intereses. 
sacroilíacas (erosiones, esclerosis, disminución del espacio intraarticular).

Un intento de consenso acordó con los hallazgos espinales, pero no en lo referente a factores constitucionales, demográficos o metabólicos requeridos para la definición de DISH $^{2,13,22-26}$.

La proliferación ósea (exostosis) y las calcificaciones vasculares son comunes en las personas mayores, pero no está claro si tienen una etiología común. Un estudio derivado del Framingham Heart Study mostró que la presencia de osteofitos en la columna lumbar y la calcificación de la aorta abdominal (CAA) se producían en los mismos individuos tras ajustar por edad y otras covariables. La inclusión de sujetos afectados por DISH (7,0\% de la muestra) no varió el análisis ${ }^{27}$. Un reciente estudio no pudo demostrar una asociación consistente entre CAA y DISH, encontrando sí una asociación significativa con edad, índice de masa corporal, perímetro abdominal y síndrome metabólico ${ }^{28}$.

Factores genéticos (COL6A1) han sido responsabilizados de un estado hiperostótico en el desarrollo de DISH y OPLL en la población japonesa ${ }^{29}$.

La causa del DISH es desconocida, pero varios factores de riesgo están implicados, en base a su frecuente asociación con diversas enfermedades metabólicas, incluyendo hiperinsulinemia, con o sin diabetes mellitus, obesidad, hiperuricemia, dislipidemia, hipertensión y el uso prolongado de isoretinol ${ }^{30-32}$.

DISH se asocia con una mayor incidencia de factores de riesgo para enfermedad coronaria y enfermedad cerebrovascular, y los pacientes con DISH tienen una probabilidad significativamente mayor de ser afectados por el síndrome metabólico que pacientes no-DISH ${ }^{33}$. Estos reportes son comunes, pero no uniformes en todos los estudios ${ }^{34,35}$.

Estos factores de riesgo comparten con la ateroesclerosis características patológicas como el daño endotelial, el depósito de lípidos y el infiltrado inflamatorio. Las lesiones valvulares tempranas son similares a los ateromas. Es un proceso biológico activo, similar a la calcificación vascular, sostenido por la tensión mecánica y la inflamación, y se asocia con la síntesis de proteínas de matriz extracelular, como las metaloproteinasas, tenascina-c, la osteopontina y la sialoproteína ósea. La coexistencia de la neoangiogénesis, la infiltración de linfocitos T, las moléculas de adhesión, y la expresión del gen HSP60 indica un proceso activo inmunomediado ${ }^{36}$.

Basados en la observación clínica del compromiso valvular aórtico en pacientes con DISH, en 2014 publicamos un trabajo cuyo objetivo principal era analizar la asociación entre la hiperostosis esquelética idiopática difusa (DISH) y la presencia de esclerosis valvular aórtica (AVS). Se evaluaron los resultados de 1000 pacientes ambulatorios consecutivos (473 varones), mayores de 50 años (promedio, 67,6 años), que habían sido examinados con un ecocardiograma Doppler y radiología torácica anterior y lateral. Globalmente, 195 pacientes (19,5\%) tuvieron diagnóstico de DISH y 283 (28,3\%) de AVS. DISH fue más prevalente que AVS en varones (66,7\% vs. $42,6 \%$, $\mathrm{p}<0,0001)$ y en pacientes de mayor edad (73,6 9 años vs. $66,1 \pm 9$ años, $\mathrm{p}<0,0001)$. El 55,4\% de los pacientes con DISH dorsal presentaron AVS vs. $21,7 \%$ de los pacientes $\sin \mathrm{DISH}(\mathrm{OR}=4,47$; IC $95 \%=3,22-6,21)$. El odds ratio (OR) ajustado por sexo y edad fue 3,04 (IC 95\%=2,12-4,36; $\mathrm{p}<0,0001)$. Se encontró una asociación estadísticamente significativa entre DISH y AVS, que se mantuvo después de ajustar por sexo y edad. La plausibilidad biológica de esta asociación se basaba en los factores de riesgo, mecanismos patogénicos y complicaciones vasculares compartidos ${ }^{37}$.

La iniciación del proceso y la relación entre las condiciones metabólicas y la neoformación ósea en el DISH son desconocidas. Se cree que esta neoformación es el resultado de un crecimiento anormal de la actividad de los osteoblastos. Este aumento se mantiene por varios factores de crecimiento que no se limitan a los huesos. Insulin-Like Growth Factor I estimula la actividad de la fosfatasa alcalina y del colágeno tipo II en los osteoblastos, y la hormona del crecimiento puede inducir la producción local de Insulin-Like Growth Factor I e Insulin-Like Growth Factor Binding Proteins en los condrocitos y los osteoblastos ${ }^{38,39}$. Debido a que la osificación se inicia en ciertos sitios, El Miedany y cols. sugieren que la hipervascularización podría ser el factor de localización del proceso ${ }^{4}$.

Algunas hormonas sistémicas, como la 1,25-hidroxivitamina $\mathrm{D}$, la hormona paratiroidea (PTH), la insulina y la leptina, y los factores de crecimiento locales, como el Transforming Growth Factor- $\beta$ (TGF- $\beta$ ), y la Bone Morphogenetic Protein (BMP), han sido estudiadas y se cree que participan en la iniciación y desarrollo de la 
osificación del ligamento vertebral (OSL) $)^{22,28,36,40}$.

La hiperleptinemia, frecuente en el sujeto obeso, contribuye al desarrollo y extensión de la calcificación del ligamento vertebral posterior ${ }^{41}$.

Un Editorial de Rheumatology concluye que el DISH podría ser considerado como parte de un síndrome más que una enfermedad, siendo entonces una expresión clínica de un desorden metabólico sistémico, para cuyo link propone la angiogénesis con un rol patogénico crucial ${ }^{42}$.

Claramente se necesitan mayores estudios para establecer los mecanismos del vínculo de asociación entre DISH y la patología vascular ${ }^{43,44}$.

Lo cierto, es que deberíamos tener en mente que esta alteración radiológica "espectacular pero inocua" puede ser un marcador importante de enfermedad metabólica sistémica.

\section{DISH en imágenes}

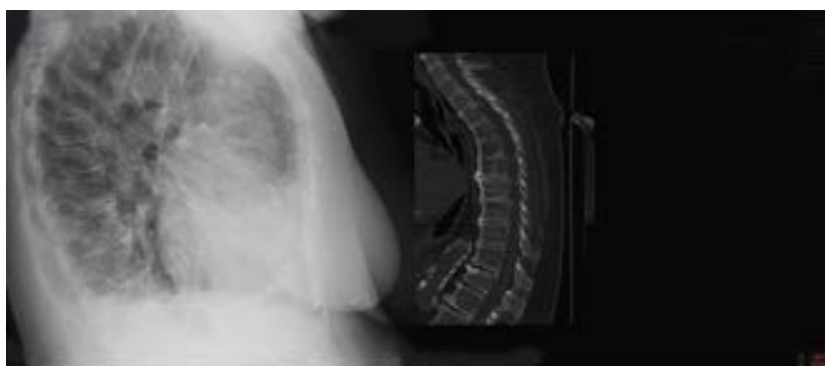

Figura 1.

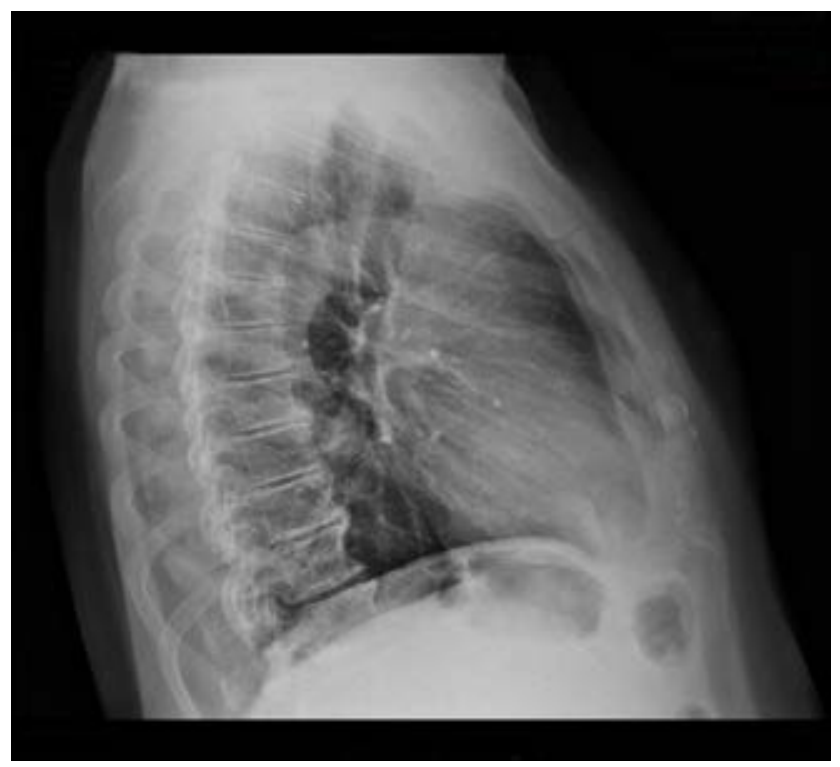

Figura 2.

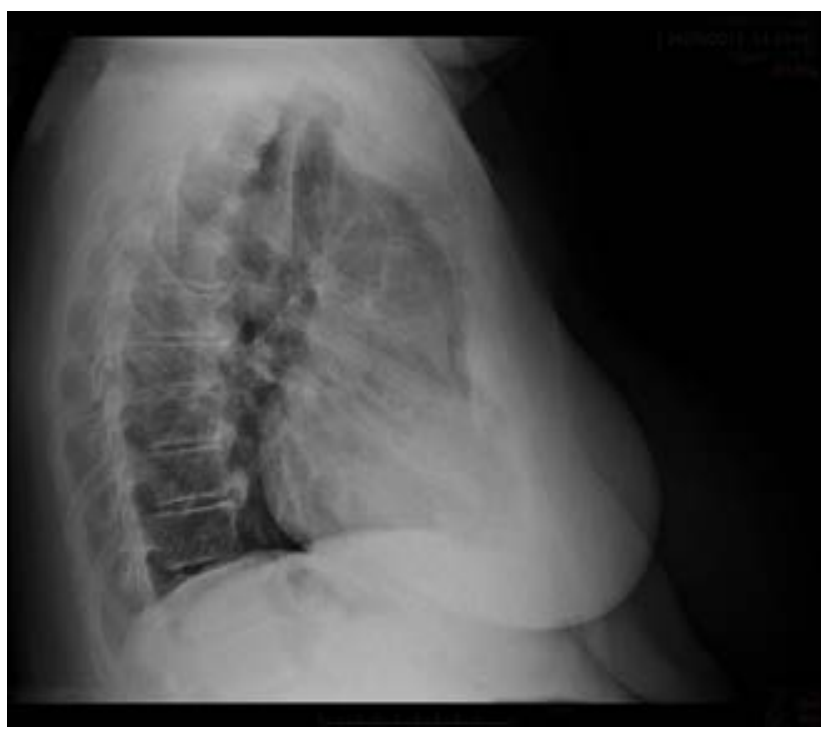

Figura 3.

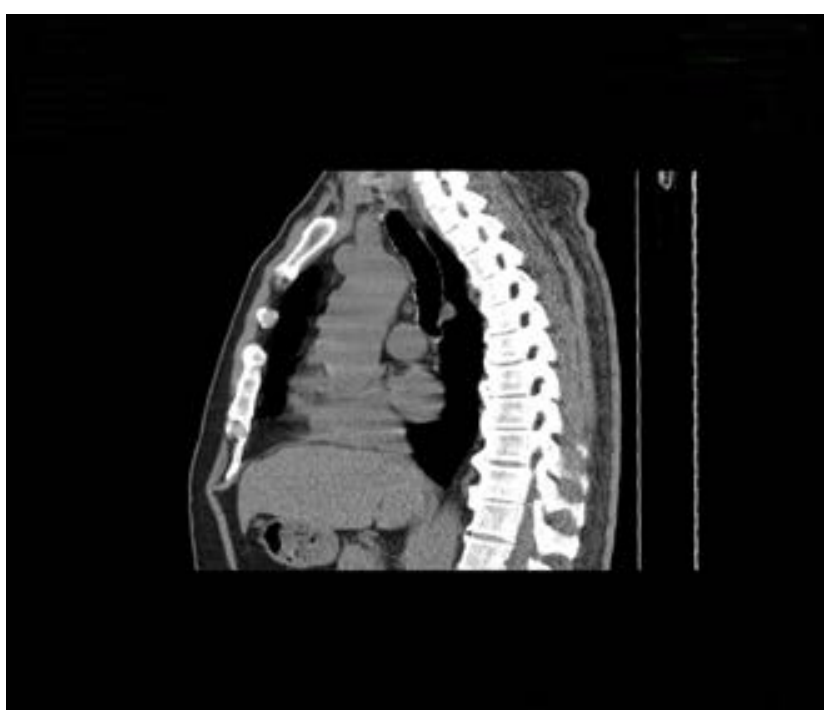

Figura 4.

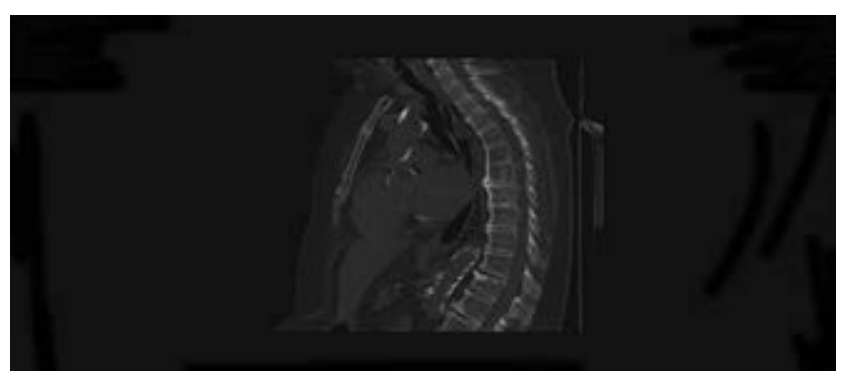

Figura 5. 


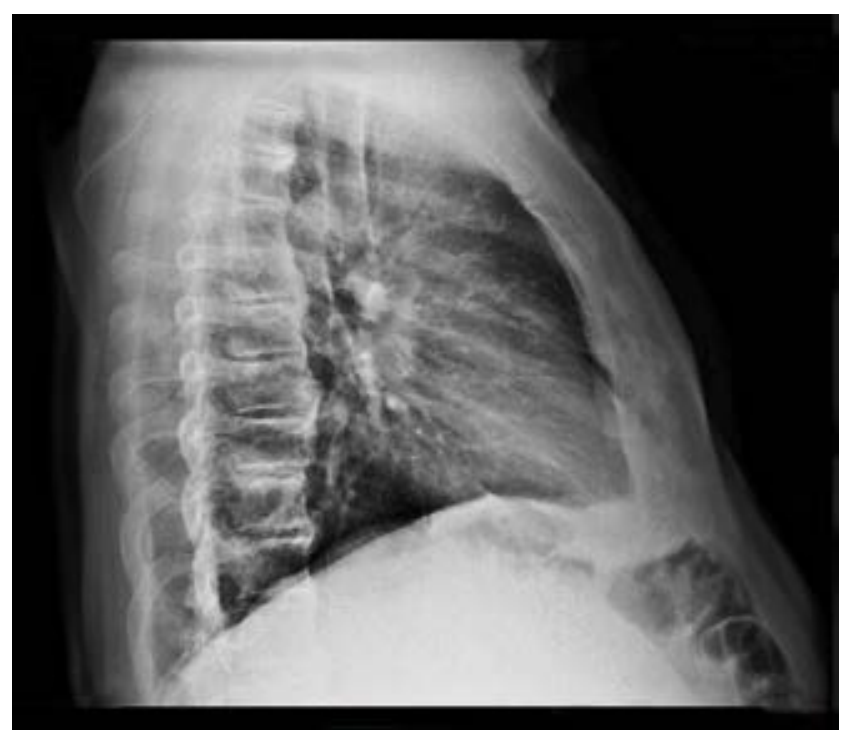

Figura 6.

\section{Bibliografía}

1. ForestierJ, Rotés-QuerolJ.Senile ankylosing hyperostosis of the spine. Ann Rheum Dis 1950; 9:321-30.

2. Resnick D, Shaul SR, Robins JM. Diffuse idiopathic skeletal hyperostosis (DISH): Forestier's disease with extraspinal manifestations. Radiology 1975; 115:513-24.

3. Epstein N. Ossification of the cervical posterior longitudinal ligament: a review. Neurosurg Focus 2002; 13:ECP1.

4. El Miedany YM, Wassif G, El Baddini M. Diffuse idiopathic skeletal hyperostosis (DISH): is it of vascular aetiology? Clin Exp Rheumatol 2000;18:193-200.

5. Mader R, Sarzi-Puttini P, Atzeni F, et al. Extraspinal manifestations of diffuse idiopathic skeletal hyperostosis. Rheumatology (Oxford) 2009; 48:1478-81.

6. Rillo OL, Scheines EJ, Moreno C, Barreira JC, Porrini AA, Maldonado Cocco JA. Coexistence of diffuse idiopathic skeletal hyperostosis and ankylosing spondylitis. Clin Rheumatol. 1989 Dec; 8(4):499-503.

7. Rotés-Querol J. Clinical manifestations of diffuse idiopathic skeletal hyperostosis (DISH). $\mathrm{Br} \quad \mathrm{J}$ Rheumatol 1996; 35:1193-4.

8. Castellano DM, Sinacori JT, Karakla DW. Stridor and dysphagia in diffuse idiopathic skeletal hyperostosis (DISH). Laryngoscope 2006; 116:341-4.

9. Ebo DG, Uytterhaegen PJ, Lagae PL, et al. Choking, sore throat with referred otalgia and dysphagia in a patient with diffuse idiopathic skeletal hyperostosis (DISH). Acta Clin Belg 2005; 60:98-101.

10. Sreedharan S, Li YH. Diffuse idiopathic skeletal hyperostosis with cervical spinal cord injury -a report of 3 cases and a literature review. Ann Acad Med Singapore 2005; 34:257-61.

11. Lee SH. Spinal subarachnoid hematoma with hyperextension lumbar fracture in diffuse skeletal hyperostosis: a case report. Spine (Phila Pa 1976) 2009; 34:E673-6.

12. Kim SK, Choi BR, Kim CG, et al. The prevalence of diffuse idiopathic skeletal hyperostosis in Korea. J Rheumatol 2004; 31:2032-5.

13. Julkunen H, Heinonen OP, Knekt P, Maatela J. The epidemiology of hyperostosis of the spine together with its symptoms and related mortality in a general population. Scand J Rheumatol 1975; 4:23-7.

14. Westerveld LA, van Ufford HM, Verlaan JJ, et al. The prevalence of diffuse idiopathic skeletal hyperostosis in an outpatient population in The Netherlands. J Rheumatol 2008; 35:1635-8.

15. Pappone N, Lubrano E, Esposito-del Puente A, et al. Prevalence of diffuse idiopathic skeletal hyperostosis in a female Italian population. Clin Exp Rheumatol 2005; 23:123-4.

16. Kiss C, O’Neill TW, Mituszova M, et al. Prevalence of diffuse idiopathic skeletal hyperostosis in Budapest, Hungary. Rheumatology (Oxford) 2002; 41:1335-6.

17. Weinfeld RM, Olson PN, Maki DD, et al. The prevalence of diffuse idiopathic skeletal hyperostosis (DISH) in two large American Midwest metropolitan hospital populations. Skeletal Radiol 1997; 26:222-5.

18. Boachie-Adjei O, Bullough PG. Incidence of ankylosing hyperostosis of the spine (Forestier's disease) at autopsy. Spine 1987; 12:739-43.

19. Cassim B, Mody G, Rubin D. The prevalence of diffuse idiopathic skeletal hyperostosis in African blacks. Br J Rheumatol 1990; 29:131-2.

20. Bloom RA. The prevalence of ankylosing hyperostosis in a Jerusalem population--with description of a method of grading the extent of the disease. Scand J Rheumatol 1984; 13:181-9.

21. Julkunen H, Knekt P, Aromaa A. Spondylosis deformans and diffuse idiopathic skeletal hyperostosis (DISH) in Finland. Scand J Rheumatol 1981; 10:193203.

22. Mazières B. Diffuse idiopathic skeletal hyperostosis (Forestier-Rotés-Querol disease): What's new? Joint 
Bone Spine 2013 Apr 5. doi: 10.1016/j.jbspin.2013 .02 .011 .

23. Utsinger PD. Diffuse idiopathic skeletal hyperostosis. Clin Rheum Dis 1985;11:325-51.

24. Mader R, Buskila D, Verlaan JJ, et al. Developing new classification criteria for diffuse idiopathic skeletal hyperostosis: back to square one. Rheumatology (Oxford) 2013; 52:326-30.

25. Resnick D, Niwayama G. Radiographic and pathologic features of spinal involvement in diffuse idiopathic skeletal hyperostosis (DISH). Radiology 1976; 119:559-68.

26. Arlet J, Mazieres B. La maladie hyperostosique. Rev Med Interne 1985; 6:553-64.

27. Karasik D, Kiel DP, Kiely DK, et al. Abdominal aortic calcification and exostoses at the hand and lumbar spine: the Framingham Study. Calcif Tissue Int 2006; 78:1-8.

28. Pariente-Rodrigo E, Sgaramella GA, Olmos-Martínez JM et al. Relationship between diffuse idiopathic skeletal hyperostosis, abdominal aortic calcification and associated metabolic disorders: Data from the Camargo Cohort. Med Clin (Barc). 2017 Mar 7. [Epub ahead of print]

29. Tsukahara S, Miyazawa N, Akagawa $\mathrm{H}$ et al. COL6A1, the candidate gene for ossification of the posterior longitudinal ligament, is associated with diffuse idiopathic skeletal hyperostosis in Japanese. Spine (Phila Pa 1976). 2005 Oct 15; 30(20):2321-4.

30. Mader R, Lavi I. Diabetes mellitus and hypertension as risk factors for early diffuse idiopathic skeletal hyperostosis (DISH). Osteoarthritis Cartilage 2009; 17:825-8.

31. Kiss C, Szilágyi M, Paksy A, et al. Risk factors for diffuse idiopathic skeletal hyperostosis: a case-control study. Rheumatology (Oxford) 2002; 41:27-30.

32. Sencan D, Elden H, Nacitarhan V, Sencan M, Kaptanoglu E. The prevalence of diffuse idiopathic skeletal hyperostosis in patients with diabetes mellitus. Rheumatol Int 2005; 25:518-21.

33. Mader R, Novofestovski I, Adawi M, et al. Metabolic syndrome and cardiovascular risk in patients with diffuse idiopathic skeletal hyperostosis. Semin Arthritis Rheum 2009; 38:361-5.

34. Mader R, Dubenski N, Lavi I. Morbidity and mortality of hospitalized patients with diffuse idiopathic skeletal hyperostosis. Rheumatol Int 2005; 26:132-6.

35. Burner TW, Rosenthal AK. Diabetes and rheumatic diseases. Curr Opin Rheumatol 2009; 21:50-4.

36. Berthelot JM, Le Goff B, Maugars Y. Pathogenesis of hyperostosis: a key role for mesenchymatous cells? Joint Bone Spine. 2013 Dec; 80(6):592-6.

37. Orden AO, David JM, Díaz RP, Nardi NN, Ejarque AC, Yöchler AB. Association of diffuse idiopathic skeletal hyperostosis and aortic valve sclerosis. Medicina (B Aires). 2014; 74(3):205-9.

38. Denko CW, Malemud CJ. Role of the growth hormone/ insulin-like growth factor-1 paracrine axis in rheumatic diseases. Semin Arthritis Rheum 2005; 35:24-34.

39. Denko CW, Malemud CJ. Body mass index and blood glucose: correlations with serum insulin, growth hormone, and insulin-like growth factor-1 levels in patients with diffuse idiopathic skeletal hyperostosis (DISH). Rheumatol Int 2006; 26: 292-7.

40. Atzeni F, Sarzi-Puttini P, Bevilacqua M. Calcium deposition and associated chronic diseases (atherosclerosis, diffuse idiopathic skeletal hyperostosis, and others). Rheum Dis Clin North Am 2006; 32:413-26.

41. Ikeda Y, Nakajima A, Aiba A et al. Association between serum leptin and bone metabolic markers, and the development of heterotopic ossification of the spinal ligament in female patients with ossification of the posterior longitudinal ligament. Eur Spine J. 2011 Sep; 20(9):1450-8.

42. Pappone N, Ambrosino P, Di Minno MN et al. Is diffuse idiopathic skeletal hyperostosis a disease or a syndrome? Need for studies on angiogenesisstimulating activity. Rheumatology (Oxford). 2016 Dec 24. [Epub ahead of print].

43. Zincarelli C, Iervolino S, Di Minno MN et al. Diffuse idiopathic skeletal hyperostosis prevalence in subjects with severe atherosclerotic cardiovascular diseases. Arthritis Care Res (Hoboken). 2012 Nov; 64(11):1765-9.

44. Pillai S, Littlejohn G. Metabolic factors in diffuse idiopathic skeletal hyperostosis - a review of clinical data. Open Rheumatol J. 2014 Dec 19; 8:116-28. 